\title{
Analysis of Spatial Variation in the Atrial Fibrillation Frequency from the Multi-channel Magnetocardiogram
}

\author{
P Langley, U Steinhoff, L Trahms, M Oeff, A Murray \\ Freeman Hospital \& Newcastle University, Newcastle upon Tyne, UK \\ Physikalisch-Technische Bundesanstalt \& Freie Universität, Berlin, Germany \\ Abstract \\ independent sources within data sets.
}

\begin{abstract}
During a magnetocardiography (MCG) study, a patient was recorded while in atrial fibrillation $(A F)$, and we used this opportunity to conduct a pilot study of the spatial characteristics of the AF signal.

Forty nine MCG channels were recorded. Atrial activity was isolated by the selection of a QRST-free section of AF. Fourier analysis identified the frequency characteristics of the atrial signal. Principal component analysis (PCA) was used to identify independent components in the atrial signal.

Several discrete frequency peaks in the range 4 to 9 $\mathrm{Hz}$ were observed which were spatially grouped. PCA separated these frequencies into discrete components.

The characteristics of the atrial signals may be associated with different re-entrant pathways.
\end{abstract}

\section{Introduction}

$\mathrm{AF}$ is a common arrhythmia affecting the elderly population. Multiple re-entrant pathways are the mechanism thought to be responsible for sustaining the arrhythmia [1]. Spatial information, necessary for therapeutic ablation, is obtained during invasive intracardiac electrophysiology studies. A simpler noninvasive technique for studying the spatial details of AF would be of great value. Spatial resolution from the 12lead ECG is very poor, but mapping of body surface potentials and magnetic fields close to the heart provide better spatial resolution [2]. Magnetic field measurements have the advantage that they are noncontact and provide more detailed information [3]. AF is difficult to study non-invasively because of the small size of the atrial signal. Techniques for the separation of the atrial signal from the large ventricular activations from body surface recordings have been developed [4]. Alternatively, QRST-free sections of lead data may be used. PCA is a statistical signal processing technique which is widely used to represent multi-variable data using a minimum data set and for identification of

\section{Methods}

\subsection{MCG recording}

The MCG of a patient in atrial fibrillation was recorded in a magnetically shielded room using a 49 sensor MCG system at the Freie Universität, Berlin. The sample rate was $1000 \mathrm{~Hz}$ and amplitude resolution was $0.01 \mathrm{pT}$. The sensor array covered an area of $21 \mathrm{~cm}$ centred $12.5 \mathrm{~cm}$ below the manubrium sterni at a height of approximately $4 \mathrm{~cm}$ above the chest, with the patient supine. Figure 1 shows schematically the sensor locations relative to the patient.

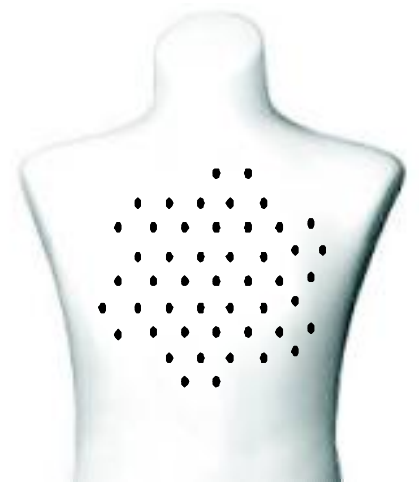

Figure 1. MCG sensor array

\subsection{Analysis}

The MCG recording was reviewed visually to identify the longest period of ventricular inactivity by the absence of QRS and T waves across the 49 channels. This section was extracted for further analysis. The longest section was chosen to provide the best frequency resolution for the subsequent frequency analysis of the atrial signal. The amplitude of the atrial signal was quantified by the peak to peak amplitude in each channel. 
Fourier analysis was used to determine the frequency content of the atrial signal in each channel with frequency resolution of approximately $1 \mathrm{~Hz}$. The region of interest is in the range 2 to $15 \mathrm{~Hz}$ since atrial fibrillation is known to lie within this range. The locations and the frequencies of the atrial signals with the largest spectral power, representing the dominant signal, were identified.

Maps of the spatial distributions of dominant peaks in the frequency spectra were plotted to provide an indication of the degree of spatial organization of the dominant frequency distribution.

PCA was applied to the atrial signals resulting in the atrial activity being represented by a substantially reduced set of signals compared to the original 49 signals. PCA models the multi-channel data by linear combinations of the data as:

$$
\mathrm{X}_{\mathrm{pca}}=\mathrm{C} * \mathrm{X}_{\text {data }}
$$

The coefficient matrix $\mathrm{C}$ is determined by eigenvectors of the covariance of data matrix $\mathrm{X}_{\mathrm{data}}$, arranged so that the corresponding eigenvalues are in descending order. Then the first principal component contains the most dominant features across the data, and subsequent components contain less dominating features.

Fourier analysis was applied to the first few principal components (these are the components containing the atrial activities) and these were compared with the analysis of the spectra of the original MCG atrial activities. Additionally, maps of the PCA coefficients were plotted, providing an indication of the contribution of each signal to the principal components.
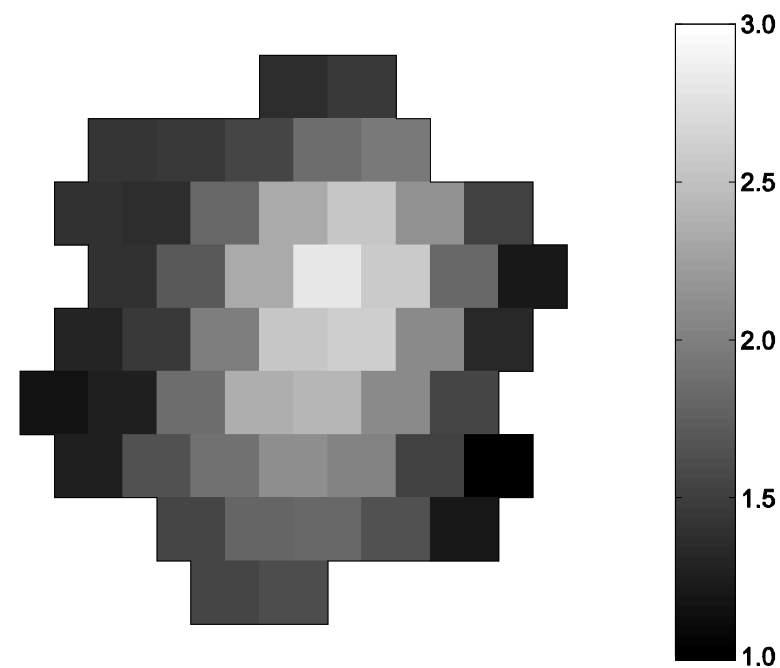

Figure 2. Atrial signal amplitude across the measurement area $(\mathrm{pT})$.

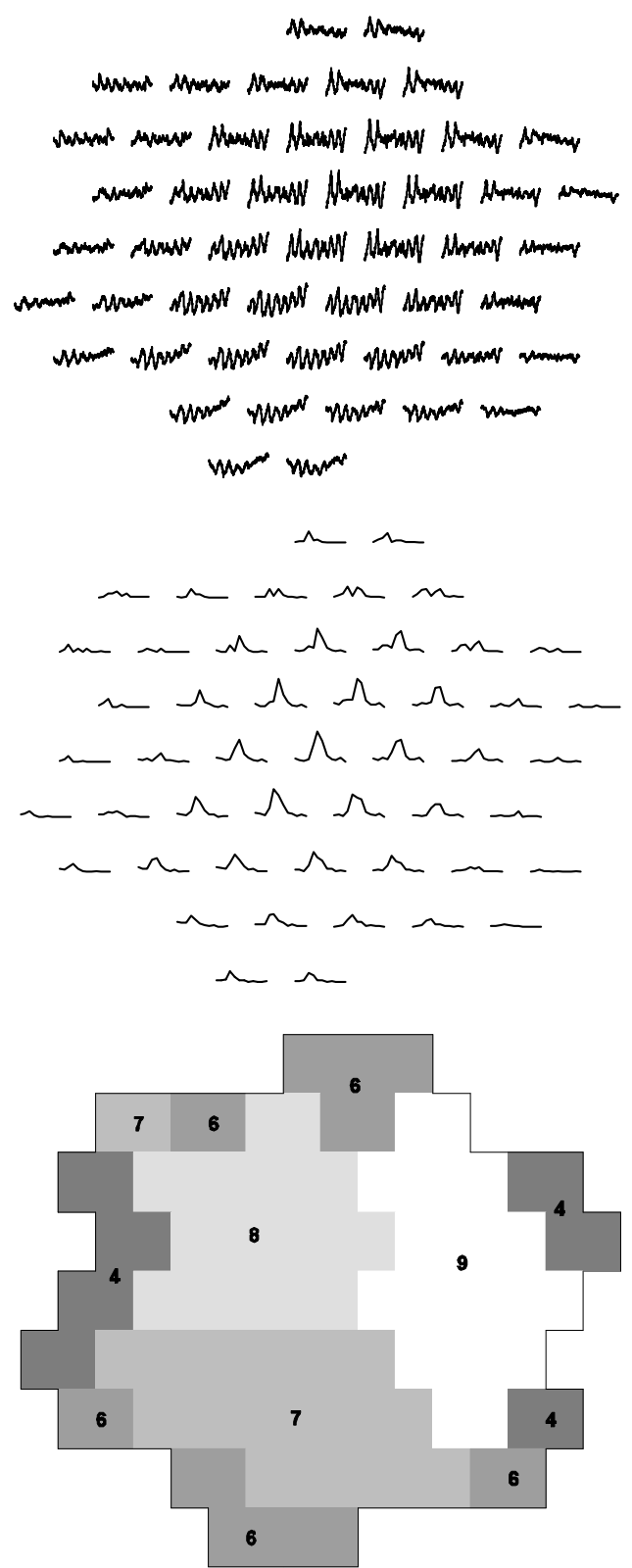

Figure 3. Atrial signals (1 s duration, maximum peak to peak amplitude $2 \mathrm{pT}$ ), power spectra $(2$ to $15 \mathrm{~Hz})$ and peak atrial frequency $(\mathrm{Hz})$ at each sensor location for the extracted atrial section. 


\section{Results}

\subsection{Atrial signal}

The section extracted for analysis had duration of $1 \mathrm{~s}$. There were variations in the amplitudes of the atrial signals across the 49 channels and these are shown in figure 2. The lighter area running from upper right (upper left chest) to lower left (lower right chest) indicating the regions of greatest amplitude. Figure 3 (top) shows the atrial section with the positions of the atrial signals corresponding to the sensor locations.

\subsection{Frequency analysis}

There were variations in the frequency content of the atrial signals across sensor locations. Across the 49 channels there were several discrete frequency peaks in the spectra ranging from 4 to $9 \mathrm{~Hz}$. Figure 3 (middle) illustrates the spectra for the atrial signals shown in figure 3 (top). The different frequency peaks were spatially grouped. Figure 3 (bottom) illustrates variations in the spatial distribution of the peak frequencies.

\subsection{Principal component analysis}

The dominant atrial activity of the multi-channel data could be represented by the first few principal components (PCs), with the remaining components containing very low level signals and noise. These PCs separated the discrete frequencies of the frequency analysis of the multi-channel data. Figure 4 shows an example of the PCA analysis. Shown are the time series plots, frequency spectra and coefficients maps of the first three PCs of the atrial section, for which the multichannel analysis is shown in figure 3. A peak in the frequency spectrum of the first PC corresponded to the peak at $8 \mathrm{~Hz}$ in the signals with the largest atrial amplitude. The coefficients map shows that the largest contribution to the first PC was from the signals in the central location of the measurement area. The second and third PCs have frequencies of 6 and $4 \mathrm{~Hz}$ respectively with maps showing contributions from the sensors at the periphery of the measurement area, in agreement with the map shown in figure 3 .
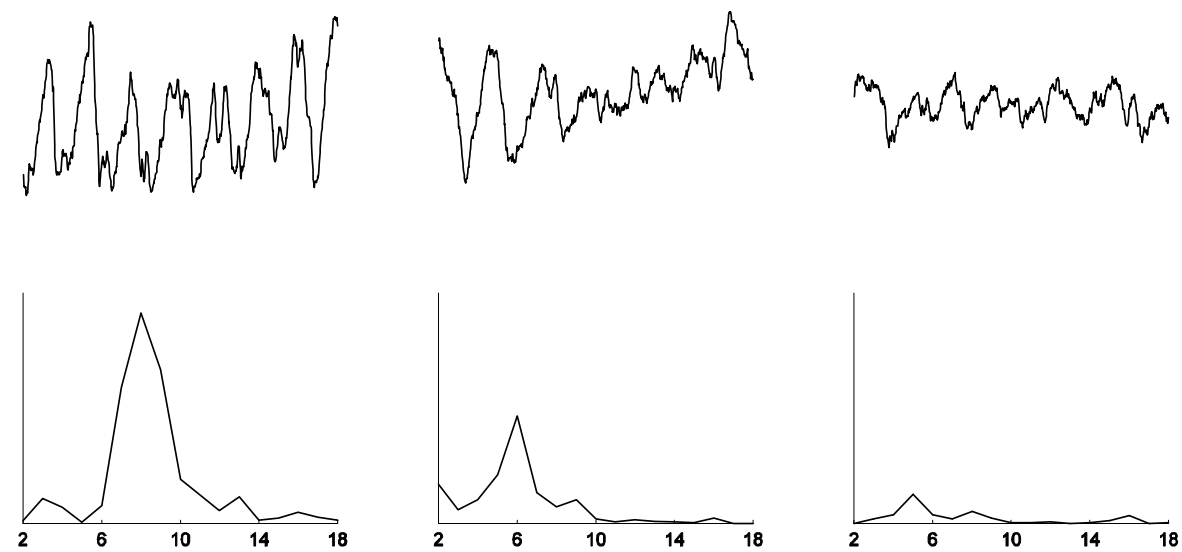

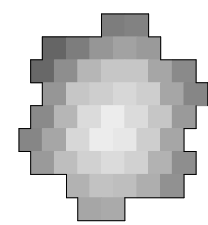

1

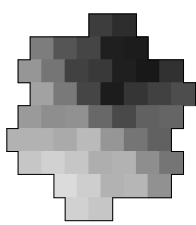

2

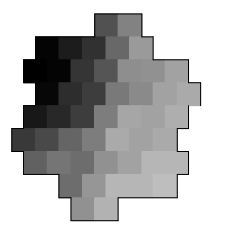

3

Figure 4. Principal component time series, spectra and coefficients maps for PCs 1,2 and 3 for the atrial section (1 s duration). PCs are plotted on the same amplitude scale with peak to peak amplitude of PC1 of $10 \mathrm{pT}$. The peaks in the spectra $(2$ to $18 \mathrm{~Hz}$ ) are at $8 \mathrm{~Hz}, 6 \mathrm{~Hz}$ and $4 \mathrm{~Hz}$ for PCs 1,2 and 3 respectively. The coefficients maps have values ranging from -0.2 (black) to +0.2 (white). 


\section{Discussion}

Atrial fibrillation is thought to be sustained by reentry. Characteristics of the re-entrant circuits contributing to the arrhythmia are expected to be reflected in the non-invasive measurements of the cardiac electric and magnetic activity. Our results show that there are variations in the amplitude and frequency content of the multi-channel MCG that are spatially organized. Differences in amplitude across the thorax are probably related to differences in distances from the cardiac source. The number of discrete frequencies observed may relate to the number of active re-entrant pathways and PCA was able to separate these signals. The frequency may relate to pathway length. In order to test these hypotheses it will be necessary to extend this pilot study. The application of atrial signal extraction techniques to separate longer periods of atrial activity will allow more detailed analysis of the dynamics of atrial fibrillation.

\section{Acknowledgements}

P Langley is supported by a research grant from the Engineering and Physical Sciences Research Council.

\section{References}

[1] Konings KT, Kirchhof CJ, Smeets JR, Wellens HJ, Penn OC, Alessie MA. High-density mapping of electrically induced atrial fibrillation in humans. Circulation 1994;89:1665-80.

[2] Lian A, Li G, Cheng J, Avitall B, He B. Body surface Laplacian mapping of atrial depolarisation in healthy human subjects. Med Biol Eng Comput 2002;40:650-9.

[3] Kosch O, Steinhoff U, Jazbinsek V, Trontelj Z, Trahms L. Non-invasively measured cardiac magnetic field maps improve the estimation of the current distribution. Comp Cardiol 2001;28:285-8

[4] Langley P, Stridh M, Rieta JJ, Sörnmo L, Millet-Roig J, Murray A. Comparison of atrial rhythm extraction techniques for the estimation of the main atrial frequency from the 12-lead electrocardiogram in atrial fibrillation. Comp Cardiol 2002;29:29-32.

Address for correspondence.

Philip Langley

Medical Physics Department

Freeman Hospital

Newcastle upon Tyne

NE7 7DN

UK

philip.langley@ncl.ac.uk 